tolerance by decreasing the number of functional receptors on the cell surface has discouraged drug discovery programmes from investigating new agonists to promote endocytosis. However, this study indicates that agonists that promote endocytosis of MOR might provide analgesics with improved tolerance profiles. In the meantime, administration of drugs that promote endocytosis of MOR with morphine might produce less tolerance than morphine alone.

Melanie Brazil Associate Editor,

Nature Reviews Drug Discovery

(2) References and links ORIGINAL RESEARCH PAPER He, L. et al. Regulation of opioid receptor trafficking and morphine tolerance by receptor oligomerization. Cell 108, 271-282 (2002)

FURTHER READING Williams, J. T. et al.

Cellular and synaptic adaptations mediating opioid dependence. Physiol. Rev. 81, 299-343 (2001) WEB SITES Whistler's laboratory:

http://www.egcrc.org/pis/whistler-r.htm Encyclopedia of Life Sciences: http://www.els.net

opiates | opiate receptors

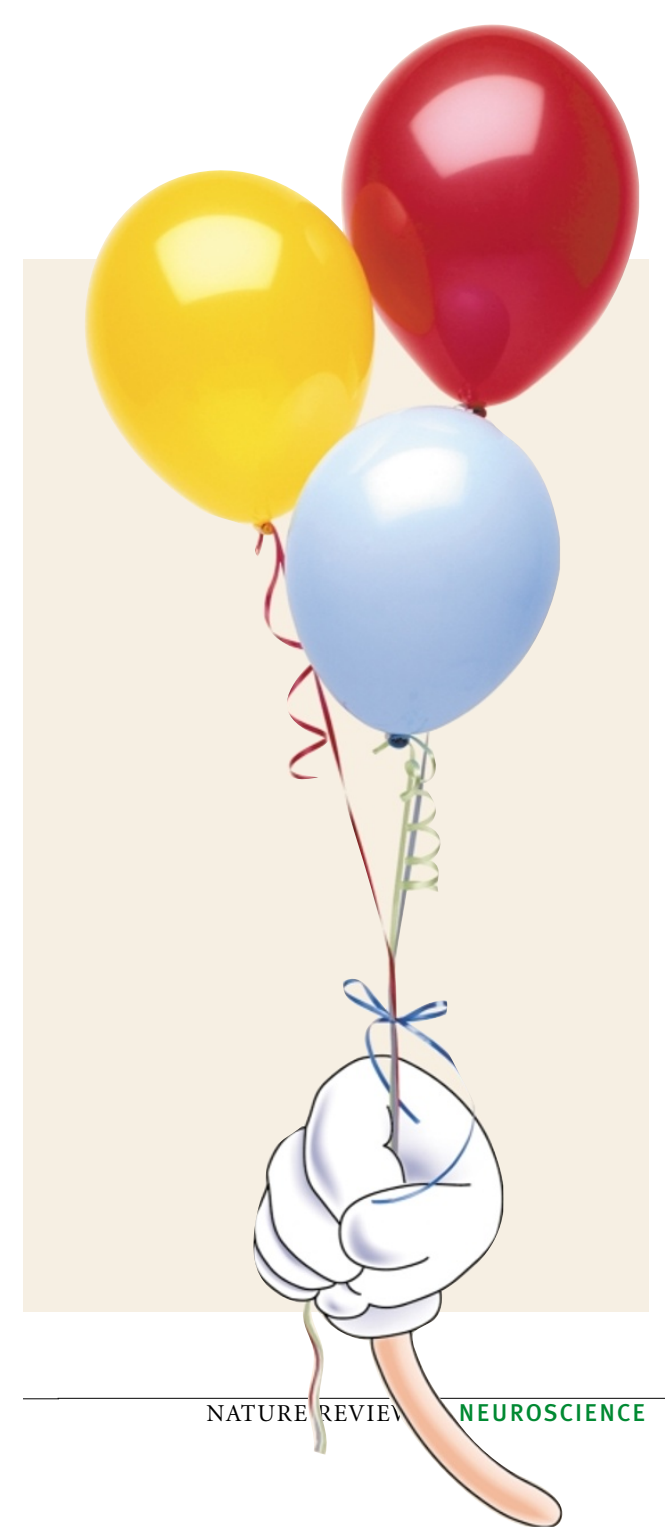



ELECTROENCEPHALOGRAPHY

\section{Brain waves in phase}

Cognitive neuroscience is making increasing use of techniques such as functional magnetic resonance imaging and electroencephalography (EEG) to visualize changes in brain activity that occur in response to particular stimuli. Individual EEGs are typically averaged to give a stimulus-evoked eventrelated potential (ERP). It is often assumed that the ERP represents the sum of individual events that occur with a fixed latency and polarity, independently of the rest of the EEG, and that the averaging process removes background EEG activity, which is considered to be noise. An alternative explanation, however, is that the ERP arises because of 'phase resetting' of ongoing EEG activity that results from the stimulus presentation.

It is becoming clear that if we are to interpret this kind of study correctly, we need to know more about the origin of the EEG and ERP. Makeig and colleagues have attempted to resolve the question of what the ERP actually represents by undertaking a detailed analysis of EEG and ERP data from a straightforward experiment in which subjects viewed simple visual stimuli that required no response.

First, they compared the averaged ERP in the period immediately after stimulus presentation with the individual EEG readings (epochs) from which it had been obtained. There were large poststimulus increases in the alpha band (between 8 and $12 \mathrm{~Hz}$ ) in the ERP waveforms that were not paralleled by similar increases in the individual EEGs. In fact, the amplitude of the ERP, at a range of frequencies below $20 \mathrm{~Hz}$, was much larger than would be expected if the phases of the individual EEG epochs were randomly distributed.

Makeig et al. then looked at the intertrial coherence, which measured how consistent the EEG phase was across trials. Before and after the stimulus presentation the phase distribution was uniform across trials, but during stimulus presentation a dominant phase distribution emerged, indicating that the phases of the EEGs had been reset by the stimulus. This phase resetting was sufficient to account for the peristimulus ERP.

Further analysis of the individual trials showed that for those trials with the lowest post-stimulus signal in the alpha band, the average ERP was also low. This is inconsistent with the commonly made assumption that the ERP sums fixed-latency, fixedpolarity events that occur independently of the rest of the EEG.

Makeig et al. next used independentcomponent analysis, which can separate out the different electrical activities that contribute to the EEG recording, to find out which portions of the EEG signals were phase reset. The results indicated that multiple independent processes, probably occurring within compact cortical domains, contribute to the recorded EEGs. The waveforms seem to result from the sum of these ongoing, independent processes, which are partially reset by the occurrence of a visual stimulus to produce the averaged ERP.

The authors comment that, in general, averaged ERPs might result from a combination of phase resetting of ongoing EEG activity with concurrent energy increases, but point out that phase resetting explains many features of the data. Given the recent surge of interest in the role of brain dynamics and correlated activity in cognitive processes, studies such as this will make an important contribution to our understanding of cortical function.

Rachel Jones

(2) References and links

ORIGINAL RESEARCH PAPER Makeig, S. et al. Dynamic brain sources of visual evoked responses. Science 295, 690-694 (2002) FURTHER READING Salinas, E. \& Sejnowski, T. J. Correlated neuronal activity and the flow of neural information. Nature Rev. Neurosci. 2, 539-550 (2001)

WEB SITES

Computational Neurobiology Laboratory: http://www.cnl.salk.edu/ Swartz Center for Computational Neuroscience:

http://sccn.ucsd.edu/ 